\title{
Quickest Spectrum Sensing Approaches for Wideband Cognitive Radio Based On STFT and CS
}

\author{
Qi Zhao ${ }^{1 *}$, Wei Qiu ${ }^{1}$, Boxue Zhang' and Bingqian Wang ${ }^{2}$ \\ ${ }^{1}$ School of Electronic and Information Engineering, Beihang University \\ Beijing, China \\ [e-mail: \{zhaoqi, sevenine\}@buaa.edu.cn; zhangboxue.2008@163.com] \\ ${ }^{2}$ AVIC Beijing Precision Engineering Institue Aircraft Industry \\ Beijing, China \\ [e-mail: 1165335368@qq.com] \\ *Corresponding author: Qi Zhao
}

Received December 16, 2017; revised September 26, 2018; accepted October 31, 2018;

published March 312019

\begin{abstract}
This paper proposes two wideband spectrum sensing approaches: (i) method A, the cumulative sum (CUSUM) algorithm with short-time Fourier transform, taking advantage of the time-frequency analysis for wideband spectrum. (ii)method B, the quickest spectrum sensing with short-time Fourier transform and compressed sensing, shortening the time of perception and improving the speed of spectrum access or exit. Moreover, method B can take advantage of the sparsity of wideband signals, sampling in the sub-Nyquist rate, and it is more suitable for wideband spectrum sensing. Simulation results show that method A significantly outperforms the single serial CUSUM detection for small SNRs, while method B is substantially better than the block detection based spectrum sensing in small probability of the false alarm.
\end{abstract}

Keywords: Quickest detection, CUSUM, Spectrum sensing, STFT, Compressed sensing 


\section{Introduction}

$\mathbf{W}_{\text {ith the huge demand for the smart phones and mobile devices, how to offload heavy }}$ traffics is a hot topics of fifth-generation (5G) mobile communications. In [1-2], it's proposed that femtocell combined with cognitive radio is a promising technology in 5G, but limited frequency resources have been behind their tremendous demand. To solve this problem, cognitive radio (CR) which provides higher spectrum efficiency by using vacant spectrum for communication with the guaranty of primary users (PU) has emerged. Therefore, how to increase dynamic spectrum access becomes the core question. Quickest detection is a continuous change point detection approach[3-4], which could detect the under-utilized spectrum for cognitive users (CU). Some classical detection methods, such as energy detection, matched filter detection and cyclic feature detection, are always based on block[5], while the quickest detection performs statistics on each sample which could achieve an agile spectrum sensing. In [6-7], a quickest detection framework is first introduced to investigate the delay of detection algorithms for spectrum sensing. An improved quickest spectrum sensing method, named successive refinement test, is proposed in [8], while cyclostationary feature based on quickest spectrum sensing is deployed in [9]. Collaborative quickest detection detects a certain distribution change in an ad-hoc network, where local decisions are made at all nodes [10-11]. At present, the research mainly focuses on the integration of spectrum sensing method and the framework of the fastest detection[19-20]. However, little research has been done into the multi-channel wideband quickest spectrum sensing which is a directive and effective method for spectrum access.

Based on the above discussion, this paper is to improve the efficiency of the licensed frequency band, and increase the opportunity spectrum access. We expanded two clues for wideband spectrum sensing. One, named method A, is the quickest spectrum sensing with the short-time Fourier transform (STFT). The STFT, which is used to distinguish different primary users, is simple to realize, powerful in resistance to noise, and suitable for the real-time processing of non-stationary signal. Method A realizes the multi-channel parallel sensing, which improves the efficiency of spectrum sensing. The other one, named method B, is the quickest spectrum sensing with STFT and compressed sensing (CS). The wideband spectrum includes several independent sub-bands licensed to given primary users. Usually, only a few primary users exist in the sub-bands, hence the wideband spectrum has the feature of sparsity. This kind of sparsity allows CS to be applied to wideband spectrum sensing with reduced sampling rate and increased spectrum access in time and frequency domain. Otherwise, traditional cumulative sum (CUSUM) test is employed in quickest spectrum sensing.

The rest of this paper is organized as follows. Sect.2 introduces the system model. Sect.3 describes the two improved quickest spectrum sensing approaches, and derives the relevant mathematical formulas. In Sect.4, simulations are conducted to prove the validity and efficiency of the proposed approaches. Finally, some conclusions and prospects are provided.

\section{System Model}

The quickest detection method is a dynamic process. Secondary user must quickly detect when the primary user signal appears or disappears within the authorized frequency band[9]. For this 
process, a more clear modeling approach can be used to express as follows. If the primary user is transmitting, the received signal at the cognitive user can be represented as:

$$
H_{1}: \quad y(i)=s(i)+u(i) .
$$

If the spectrum licensed to the primary user is underutilized, the received signal can be represented as:

$$
H_{0}: \quad y(i)=u(i)
$$

where $s(i) \sim \mathrm{N}(0, P), u(i) \sim \mathrm{N}\left(0, \sigma^{2}\right)$, and $i$ stands for a time parameter.

Based on this, two basic parameters for spectrum sensing can be defined: probability of detection which is the probability of determining the current state as $H_{1}$ under $H_{1}$; probability of false alarm which is the probability of determining the current state as $H_{1}$ under $H_{0}$. In order to protect the primary user, the probability of detection should be set large enough. And in order to increase the possibility of the access of the cognitive user, the probability of false alarm should be set sufficiently small.

The standard normal distribution function is defined as:

$$
Q(x)=\frac{1}{\sqrt{2 \pi}} \int_{x}^{+\infty} e^{\frac{-t^{2}}{2}} d t
$$

So the probability of false alarm is defined as:

$$
P_{f}=Q\left(\frac{\varepsilon-\sigma^{2} \sum_{k=1}^{K} g_{k}}{\sigma^{2} \sqrt{\sum_{k=1}^{K} \frac{g_{k}^{2}}{N_{k}}}}\right)=Q\left(\frac{\varepsilon-\sigma^{2} \sum_{k=1}^{K} g_{k}}{\sigma^{2} \sqrt{\frac{\tau f_{s}}{K}}}\right)
$$

where $\varepsilon$ is the threshold. Correspondingly, the probability of detection is:

$$
P_{d}=Q\left(\frac{\varepsilon-\sigma^{2} \sum_{k=1}^{K} g_{k}\left(1+\left|h_{k}\right|^{2} \gamma\right)}{\sigma^{2} \sqrt{\sum_{k=1}^{K} \frac{g_{k}^{2}}{N_{k}}\left(1+2\left|h_{k}\right|^{2} \gamma\right)}}\right)=Q\left(\frac{\varepsilon-\sigma^{2} \sum_{k=1}^{K} g_{k}\left(1+\left|h_{k}\right|^{2} \gamma\right)}{\sigma^{2} \sqrt{\frac{\tau f_{S}}{K}} \sum_{k=1}^{K} g_{k}{ }^{2}\left(1+2\left|h_{k}\right|^{2} \gamma\right)}\right)
$$

where $g_{k}$ means the weight coefficient of each user. The best $g_{k}$ is defined as:

$$
g_{k}=\frac{\left|h_{k}\right|^{2}}{\sqrt{\sum_{k=1}^{K}\left|h_{k}\right|^{4}}}
$$

where $h_{k}$ is the coefficient of each channel.

When the primary user appears at an unknown time $\tau, y(i)$ becomes:

$$
y(i)= \begin{cases}u(i) & i=1,2, \ldots \tau-1, \\ s(i)+u(i) & i=\tau, \tau+1, \ldots . .\end{cases}
$$

Following [10-11], we use the average run length of the worst-case delay $\bar{T}_{1}$ and average run length of false alarm $\bar{T}_{0}$ as the performance evaluation criteria:

$$
\bar{T}_{1}=\sup _{\tau \geq 1} \operatorname{ess} \sup E_{p_{1}}\left\{T=t-\tau \mid t \geq \tau, y_{1}^{\tau}\right\},
$$




$$
\bar{T}_{0}=E_{p_{0}}\{t\},
$$

where sup means superiority, ess means essential, and $\tau$ is when the primary user appears, however $t$ is the time for secondary users to detect. $T=t-\tau$ is defined as the detection delay, and $T<0$ is the event of false alarm. And $y_{1}^{\tau}$ is the observation sequence before the primary user appears and $E_{p_{1}}$ indicates the conditional expectation under the assumption that the primary user appears at $\tau$. [15].

Single-channel model can be extended to a broadband signal model. The received signal in the time domain can be expressed as:

$$
x(i)=s_{k}(i)+u(i)
$$

where $k$ is in order to distinguish the different primary users. According to the observation sequence in time domain, it is difficult to detect the change points of different original users. Thus, a simple method is to distinguish the different primary users in the frequency domain.

\section{Quickest Spectrum Sensing Approaches Based on STFT and CS}

\subsection{Method A}

\subsubsection{Algorithm}

STFT is described as follows. For the time domain signal, windowing is performed to realize the localized time. On the other hand, Fourier transform realized the localized frequency for the windowed signal, which is limited to the assumption of the samples to be piecewise stationary. Fig. 1 shows the details of method A. With the STFT translation of window, an intuitional time-frequency spectrum is obtained and the one-dimensional time domain signal $x(i)$ will become a two-dimensional matrix $X_{m, n}$, in which $m$ is the time sampling-point and $n$ is the frequency sampling-point. From the two-dimensional matrix $X_{m, n}$, the primary users with different carrier frequencies can be distinguished obviously, however the accuracy between the time domain and the frequency domain need a trade-off restricted by the uncertainty principle. It is not much in evidence to implement for $X_{m, n}$, since the statistics of quickest detection algorithm are inter-compared with each other:

$$
\bar{X}_{m, n}=\operatorname{Re}\left[X_{m, n}\right]+\operatorname{Im}\left[X_{m, n}\right]
$$

Cumulative sum (CUSUM) algorithm, which is a well-known quickest detection method, then obtains the change-point. For the sampling-point $\bar{X}_{m, n}$, the log-likelihood ratio at $\forall m$ and $\forall n$ can be defined as:

$$
l_{m, n}=\log \left(\frac{f_{1 n}\left(\bar{X}_{m, n}\right)}{f_{0 n}\left(\bar{X}_{m, n}\right)}\right), \quad m=1,2,3 \ldots, n=1,2,3 \ldots,
$$

where $f_{0 n}$ means the observation samples before the frequency band is occupied, the observation samples are from the Gaussian noise, the mean of $l$ has a negative drift at the same $n$, while after that, the observation samples $f_{1 n}$ are from different primary users and Gaussian noise, the mean of $l$ has a positive drift at the same $n$ :

$$
E_{f_{0 n}}\left\{l_{m, n}\right\}=\int f_{0 n}(\bar{x}) \log \left\{\frac{f_{1 n}(\bar{x})}{f_{0 n}(\bar{x})}\right\} d \bar{x}=-D\left(f_{0 n} \| f_{1 n}\right) \leq 0,
$$




$$
E_{f_{1 n}}\left\{l_{m, n}\right\}=\int f_{1 n}(\bar{x}) \log \left\{\frac{f_{1 n}(\bar{x})}{f_{0 n}(\bar{x})}\right\} d \bar{x}=D\left(f_{1 n} \| f_{0 n}\right) \geq 0,
$$

where $D\left(f_{0 n} \| f_{1 n}\right)$ is the Kullback-Leibler divergence of $f_{0 n}$ from $f_{1 n}$, and $D\left(f_{1 n} \| f_{0 n}\right)$ is defined similarly.

According to $l$, an intuitive algorithm has the change-point at each $n$ :

$$
S_{n}=\max _{n, k \leq t}\left\{\sum_{m=1}^{\bar{t}} l_{m, n}-\sum_{m=1}^{k} l_{m, n}\right\}=\max _{n, k \leq t} \sum_{m=k+1}^{\bar{t}} l_{m, n},
$$

where $\bar{t}$ is the detection time after windowing. We suppose that the final detection time is that $\bar{t}$ multiplies by the window length. After $k, s_{n}$ is the largest one among all the statistics, which can be simplified by the recursive expression:

$$
s_{m, n}=\max \left(s_{m-1, n}+l_{m, n}, 0\right),
$$

where $s_{0, n}=0$.

$$
t_{n}=\min \left\{m \mid s_{m, n} \geq \gamma\right\}
$$

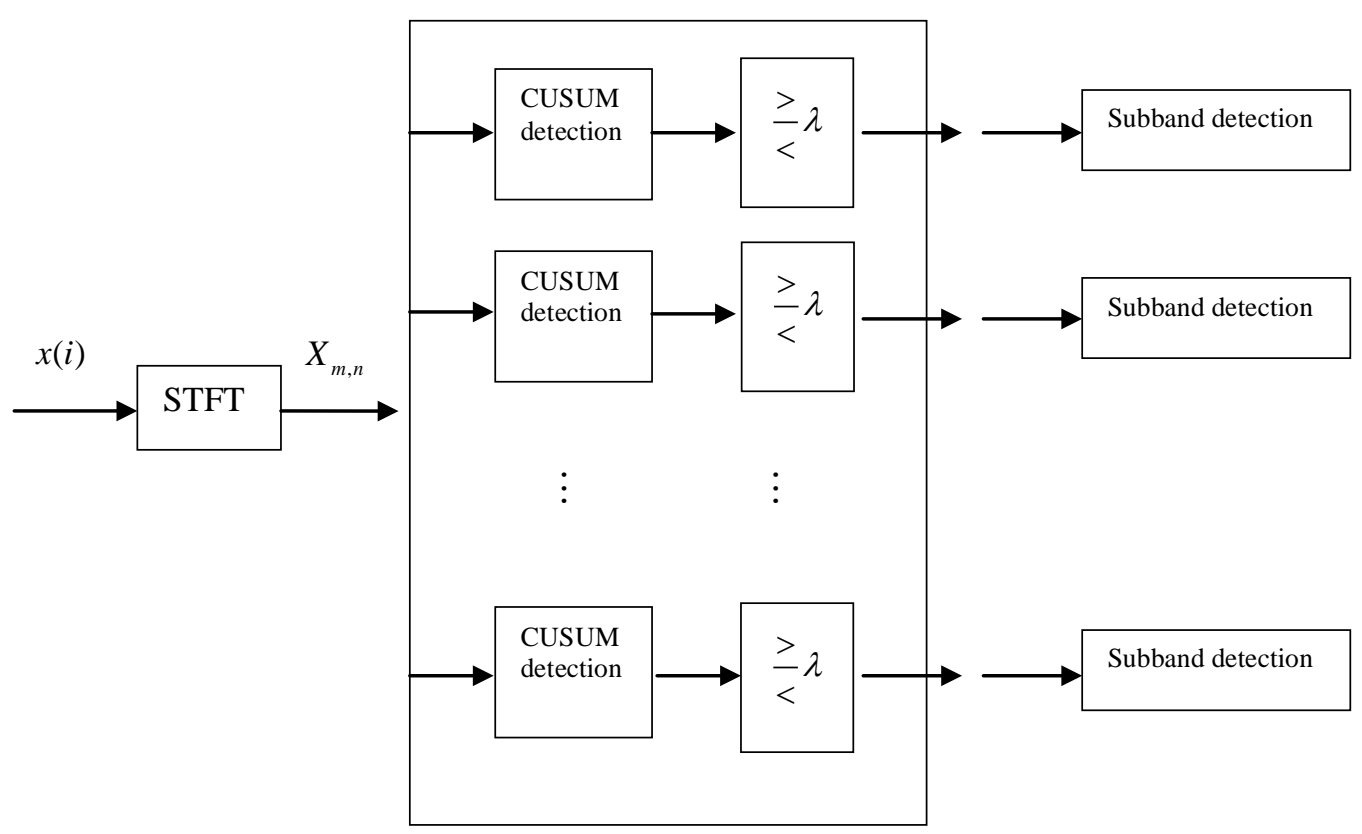

Fig. 1. The process of method A

\subsubsection{Asymptotic Performance Analyses}

Assume that STFT adopts rectangular window, the length of the window is $M$, and the length of fast Fourier transformation (FFT) for the windowed signal is also $M$.

Before STFT, the distribution sequences of the primary users satisfy: 


$$
S_{k}(i) \sim \mathrm{N}\left(0, P_{k}\right), \quad i=1,2,3, \ldots N .
$$

We can assume that the spectrum influence of the primary users with different carrier frequency would be negligible. Hence, after STFT, if the frequency band is only with the random noise,

$$
X_{m, n} \sim \mathrm{N}\left(0, M \sigma^{2}\right), m=1,2,3, \ldots \frac{N}{M}, n=1,2,3, . ., M .
$$

On the other hand, if the frequency band is the primary user with the random noise,

$$
X_{m, n} \sim \mathrm{N}\left(0, M P_{k}+M \sigma^{2}\right), \quad m=1,2,3, \ldots \frac{N}{M}, n=1,2,3, . ., M .
$$

Then if there is only random noise, we have $\bar{X}_{m, n} \sim \mathrm{N}\left(0, M \sigma^{2}\right)$. And if there is the primary user with the random noise, we have $\bar{X}_{m, n} \sim \mathrm{N}\left(0, M P_{k}+M \sigma^{2}\right)$, which can be used in Equation (12).

The log-likelihood ratio can be represent as:

$$
l_{m, n}=\frac{P_{k} \bar{X}_{m, n}^{2}}{2\left(M P_{k}+M \sigma^{2}\right) \sigma^{2}}+\frac{1}{2} \log \left\{\frac{\sigma^{2}}{P_{k}+\sigma^{2}}\right\} .
$$

We can also have:

$$
\begin{gathered}
D\left(f_{0 n} \| f_{1 n}\right)=-\frac{P_{k}}{2\left(P_{k}+\sigma^{2}\right)}-\frac{1}{2} \ln \left\{\frac{\sigma^{2}}{P_{k}+\sigma^{2}}\right\}, \\
D\left(f_{1 n} \| f_{0 n}\right)=\frac{1}{2} \ln \left\{\frac{\sigma^{2}}{P_{k}+\sigma^{2}}\right\}+\frac{P_{k}}{2 \sigma^{2}} .
\end{gathered}
$$

Therefore, following [15], the average run length of the worst-case delay $\bar{T}_{1 n}$ and average run length of false alarm $\bar{T}_{0 n}$ is approximated by

Some simple bounds on $\bar{T}_{1}$ and $\bar{T}_{0}$ can be derived:

$$
\begin{gathered}
\bar{T}_{1 n} \leq \frac{\gamma}{D\left(f_{1 n} \| f_{0 n}\right)}, \\
\bar{T}_{0 n} \geq e^{\gamma} .
\end{gathered}
$$

$$
\begin{gathered}
\bar{T}_{1 k} \leq \frac{M \gamma}{D\left(f_{1 k} \| f_{0}\right)}, \\
\bar{T}_{0} \geq M e^{\gamma} .
\end{gathered}
$$

We can compute the average run length of the worst-case delay according to given average run length of false alarm.

\subsection{Method B}

Method A improves the dynamic spectrum access, however needs a high Nyquist sampling rate under Shannon's theorem. Naturally, if the sampling frequency could be reduced, the lower energy would be needed. CS shows clearly that signals can be almost accurately reconstructed from a limited number of measurements if they are sparse or compressible, possibly contaminated with noise [13]. Therefore, the CS process, including the sparse representation of matrix, the choice of observation matrix, and the reconstruction of signal, makes the sampling frequency reduce, which can be described in more detail below: 
Firstly, if $x$ which we view as an $I \times 1$ column vector is compressible on a orthogonal basis $\Psi, x=\Psi \beta$, where $\Psi$ is the $I \times 1$ matrix and $\beta$ is the $I \times 1$ column vector. $\beta$ is considered as the equivalent sparse representation of $x$.

Secondly, the $L \times 1$ dimensional observation sequence can be shown as: $y=\Phi \beta=\Phi \Psi^{T} x$, where $(\cdot)^{T}$ denotes the matrix transpose and $\Phi$ is a stationary $L \times I$ matrix called observation matrix which is irrelevant of $\Psi$. And we set $\Gamma=\Phi \Psi^{T}$.

Finally, obtain the accurate or approximate solution of $x$, which is based on a strict mathematical optimization problem: $\min \left\|y-\Phi \Psi^{T} x\right\|_{2}+\lambda\left\|\Psi^{T} x\right\|_{0}$, where \|\|$_{2}$ and \|\|$_{0}$ is the 2-norm and 0-norm, respectively. The 2-norm is the square root of the largest characteristic value of the product of the matirix $A$ end $A^{T}$. And 0 -norm is the number of nonzero elements in the matrix.

Otherwise, restricted isometry property (RIP) indicates the sufficient and necessary condition that the determined solution exists [17]. If $\Phi$ adopts a random Gaussian matrix, it would obey RIP with high probability [18].

The sparsity in the wideband spectrum is defined as:

$$
\eta=\frac{\text { number of occupied sub-bands }}{\text { total number of sub-bands }},
$$

and the compression ratio is known as:

$$
\zeta=\frac{L}{I}
$$

Generally, $\zeta>\eta$.

The orthogonal matching pursuit (OMP) algorithm, which is a greed iteration algorithm, reconstructs the compressed signal $y$. In each iteration, the selected column $q$ from $\Gamma$ should be maximum related with the current redundancy vector $r$. Then obtain the approximate solution $\hat{x}$ of current $x$ by this column and refine the redundancy vector. Until the iteration number satisfies predetermined requirements, iteration stops. The steps of OMP algorithm are described as follows:

The initial definition: $r_{0}=y$ and iterations $i=1$, incremental matrix $A$ and $A_{0}=\varnothing$;

Firstly, the selected column $q_{i}=\underset{j=1,2, \ldots, I}{\arg \max }\left|<r_{i-1}, \Gamma_{j}>\right|$;

Then, refine $A_{i}=\left[A_{i-1}, \Gamma_{q_{i}}\right]$;

Thirdly, get the solution $\hat{x}_{i}=<\mathrm{A}_{i}, \mathrm{~A}_{i}>^{-1}\left(\mathrm{~A}_{i}\right)^{T} y$ based on the least-square method;

Finally, refine $r_{i}=y-\Gamma \hat{x}_{i}$ and $i=i+1$. Until $i$ satisfies the condition, we have $\hat{x}$ and iteration stops.

The procedure of block detection is as follows: first,the received signal is divided into several small blocks by a fixed sampling length, and it is assumed that the signals in each block are kept in the same state. We will get two species of signal, "there is primary user signal" and "no primary user signal " by this way. Then every small block is an unit, it calculates the statistics within the block and compares with a set threshold, then it gets the band of the idle situation.

Fig. 2 shows the process of method B, and after CS which is supposed to be ideal reconstruction, STFT is used for $\hat{x}$. We can have the observation sequence at the cognitive user using Equation (11). The cognitive user obtains using Equation (12) after each sample, 
computes s using Equation (16), compares the statistics at each frequency sampling point using Equation (18), and has the detection time in each frequency band. One focus is that CS will inevitably introduce error for spectrum sensing. It is unable to reach the near constant false alarm rate (CFAR) like CUSUM detection and method A. So, we employ a new system specification called the probability of false alarm which is of interest for spectrum sensing. Another performance indicator remains detection delay. Note that the threshold is the key to system performance, and it can be tuned using experimental results.

Both Method A and Method B improve the efficiency of wideband spectrum sensing from the perspective of detection approaches. Besides these, a collaborative video scheduling scheme is designed in [21]. And in [22], a totally distributed content delivery system is proposed. These two ways are mainly for device-to-device communication. In [23], we can learn an exact expression for the stochastic fluid model and characterize the performance which improve the data service for the Industrial Internet of Things.

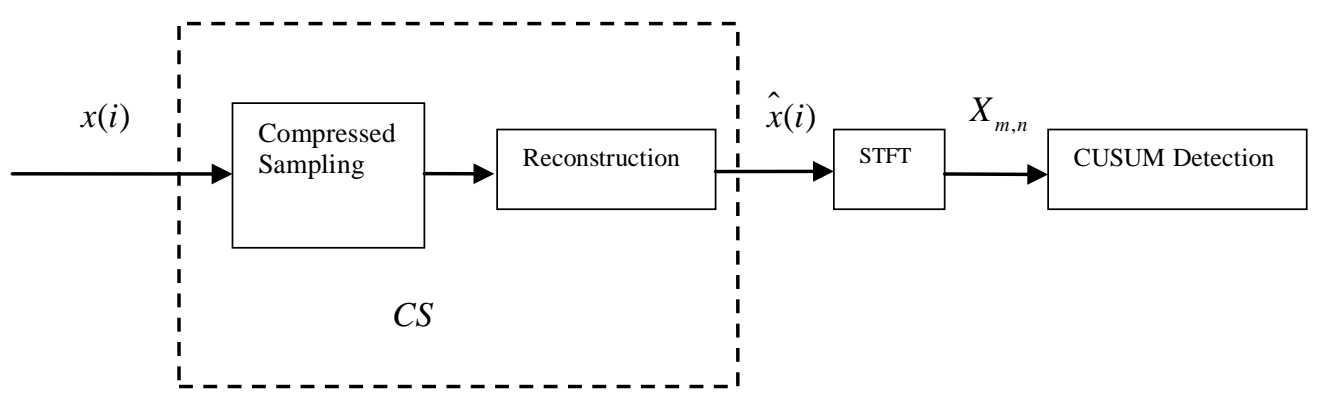

Fig. 2. The process of method B

\section{Simulations and Results}

In this section, we show the performance of wideband spectrum sensing, including method A and method B. We assume that $\sigma^{2}=1$, there are five primary BPSK modulated signals. We set the length of the window to 20 sampling points, the motion of the window is not overlapping. Detection delay is the average delay of all detection methods, serial CUSUM detection or block based spectrum sensing. The detection time is the last time, when all the primary users complete the detection task.

We first simulate the performance of method A. Fig. 3 shows the relationship between the detection delay and SNR under the same threshold. The detection delay is uniformly reduced as the SNR increases. The log-likelihood ratio deviates from zero farther for a large SNR, hence large statistics are output. Large statistics result in a small detection delay under the same threshold. For example, the detection delay decreases by about 90 percent when the SNR increases from 0.5 to 2.5 . 


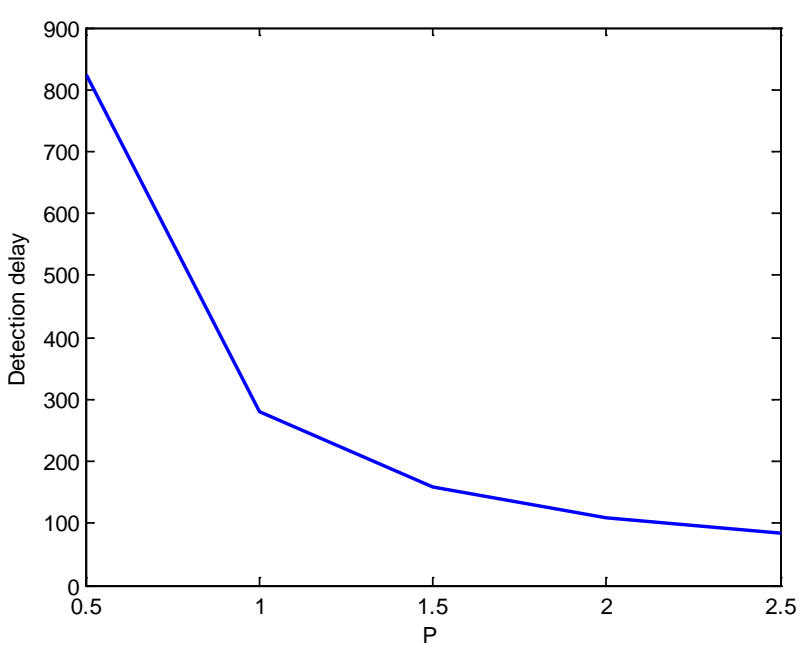

Fig. 3. Method A: detection delay versus $P$

Fig. 4 compares the distribution of the detection delay of the various algorithms when we set the same $\bar{T}_{0}$. When $\gamma=50$ for method A, the threshold is 53 for CUSUM detection. The detection delay of method A distributes centrally in some points, while the detection delay of CUSUM detection is almost discrete. When $P=2.5$, we observe that the largest percent of the detection delay is 0.4168 in method A, whereas that is 0.02714 in CUSUM detection. The distribution of the detection delay in method $\mathrm{A}$ is of an obvious advance than CUSUM detection under small $P$. For example, when $P=0.5$, the detection delay of greatest chance is 659 in method A, while that is 1104 in CUSUM detection. For the average detection delay, method A is uniformly better than the CUSUM detection for small $P$, for example, about 157.5 in method A and 180 in CUSUM detection when $P=1.5$.

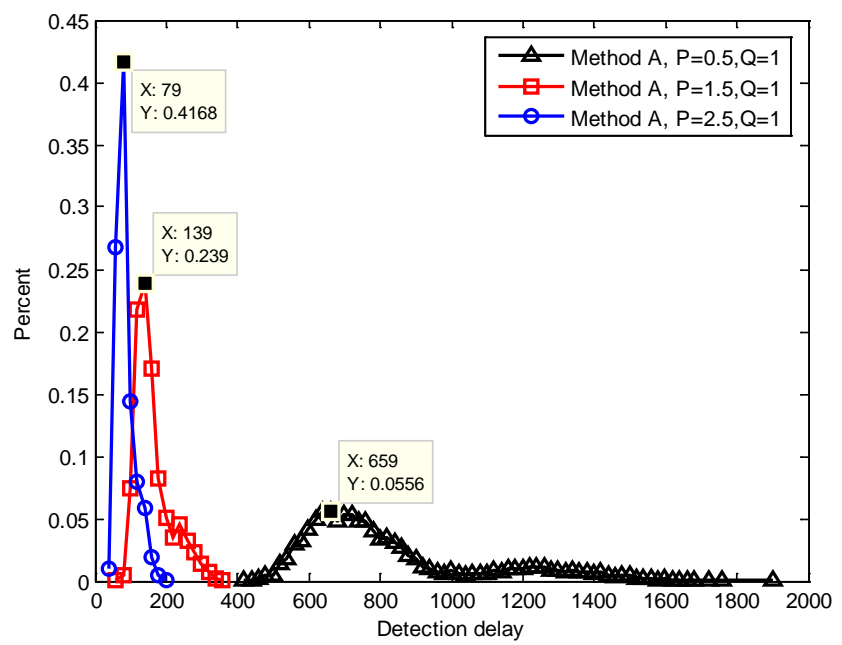

(a) 


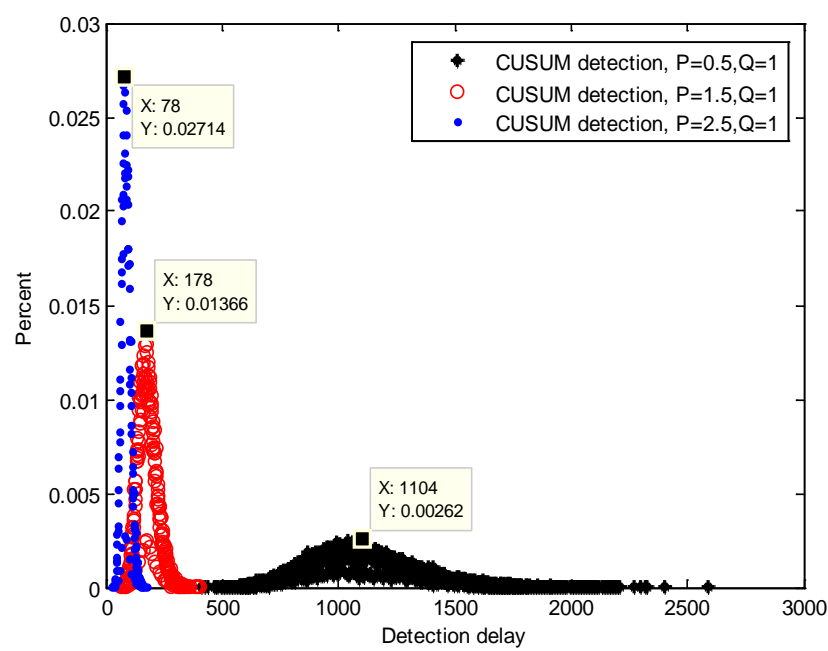

(b)

Fig. 4. Distribution of the detection delay in method A and CUSUM detection

Fig. 5 shows a comparison between method A and CUSUM detection at different $\log \left(\bar{T}_{0}\right)$ when $P=0.5$. Parallel detection time is substantially better than serial detection time. An example is that the detection time is about 2780 for CUSUM detection and about 760 for method A when $\log \left(\bar{T}_{0}\right)=\log \left(20 \mathrm{e}^{10}\right) \approx 13$.
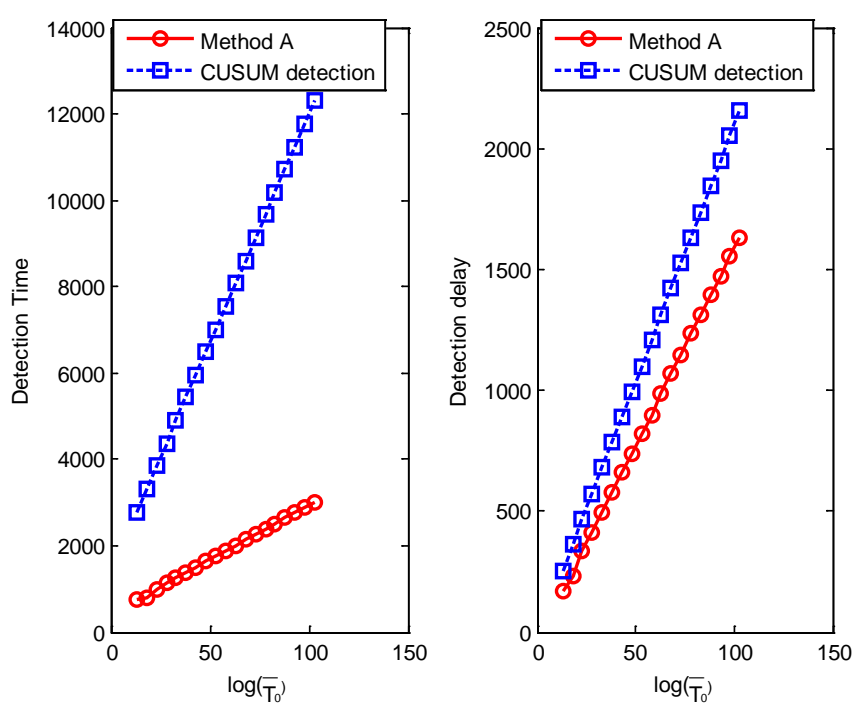

Fig. 5. Detection Time versus $\log \left(\bar{T}_{0}\right)$ and Detection delay versus $\log \left(\bar{T}_{0}\right)$ in method A and CUSUM detection when $P=0.5$

We then simulate the performance of method B. We set $\eta=0.4$, that is to say there are only two primary users in the wideband, and $\zeta=0.5$. Suppose that $P=0.5$, and the primary users start transmission at the $101^{\text {th }}$ point and $301^{\text {th }}$ point, respectively.

From Fig. 6(a), method B is superior than block detection when the probability of false alarm is more than about 0.25 which also reflects the probability of false alarms for the fastest 
detection. For example, the detection delay is around 90 for method B but around 100 for block detection when the probability of false alarm is 0.1. Otherwise, method B is quite suited to the situation that needs a small detection time as Fig. 6(b) describes.

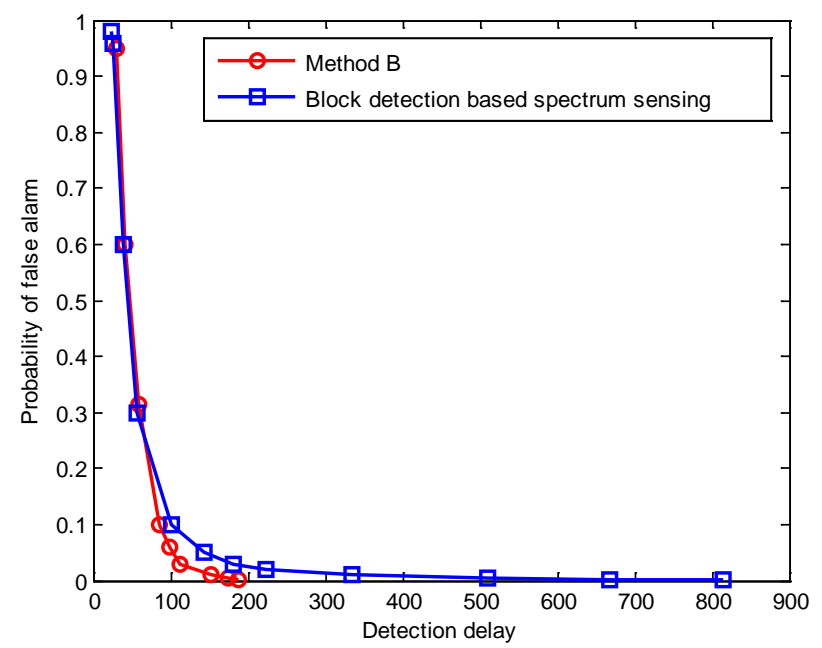

(a)

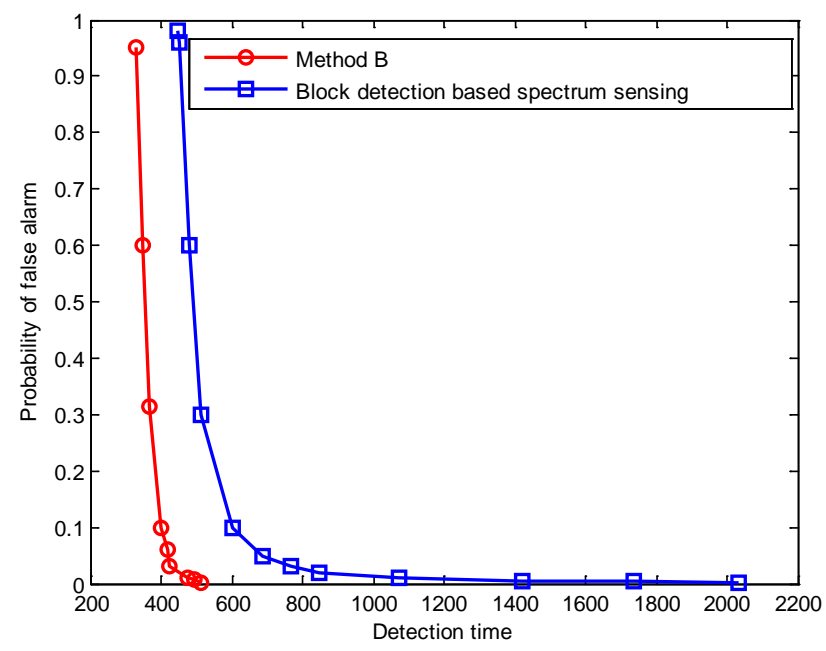

(b)

Fig. 6. Method B versus block detection based spectrum sensing

Fig. 7 shows the comparison between method A, method B and block detection. Method B was performed under the condition that the sparse degree was 0.4 and the compression rate was 0.5 . We can conclude that the detection delay of method $\mathrm{A}$ is the smallest and then that of method B. Method B is developed on the basis of A, and the main purpose is to reduce the high sampling rate when applied to wideband signals detection. Method B can take advantage of the sparsity of wideband signals, sampling in the sub-Nyquist rate for sparse representation and reconstruction of signals, and then performs quickest spectrum sensing of the reconstructed signals. The reconstructed signals will have SNR loss or signals distortion, so it can be predicted that the performance of method A is better than method B. However, both method A and B have better performance than block detection, and method B is more suitable for wideband spectrum sensing than method A. 


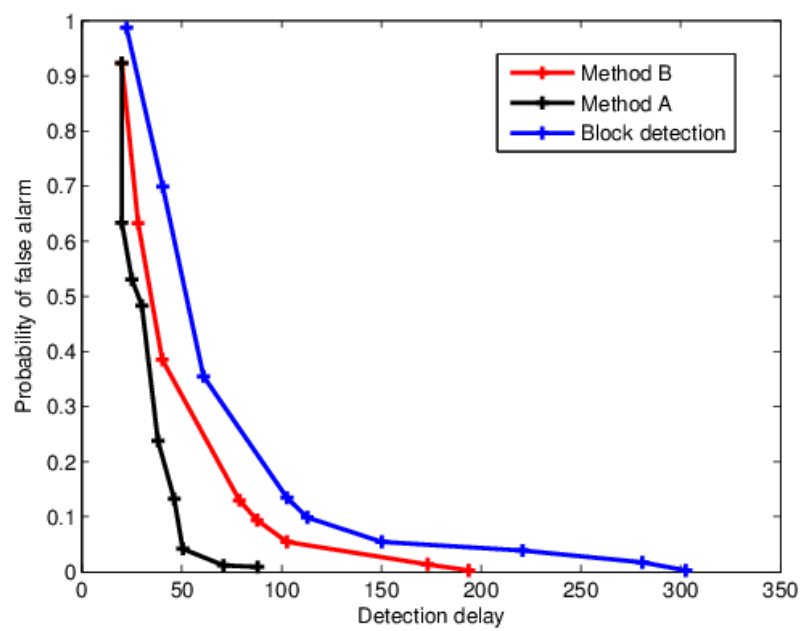

Fig. 7. Comparison between method A, method B and block detection based spectrum sensing

\section{Summary}

To improve the efficiency of wideband spectrum sensing, two novel quickest detection approaches based on STFT and CS are proposed. Compared with signal-channel serial spectrum sensing, the parallel method could substantially improve the spectrum access opportunity. Our simulations give comprehensive performance evaluation including the considerations of the detection delay, the mean time to false alarms and the probability of false alarm. Simulation results validate of the algorithms. In the future, we will study improved algorithms about the cooperative wideband spectrum sensing.

\section{References}

[1] Zhang H, Nie Y, Cheng J, et al., "Sensing Time Optimization and Power Control for Energy Efficient Cognitive Small Cell with Imperfect Hybrid Spectrum Sensing[J],” IEEE Transactions on Wireless Communications, vol. 16, no. 2, pp. 730-743, Feb. 2017. Article (CrossRef Link).

[2] Zhang H, Jiang C, Mao X, et al., "Interference-Limited Resource Optimization in Cognitive Femtocells With Fairness and Imperfect Spectrum Sensing[J],” IEEE Transactions on Vehicular Technology, vol. 65, no. 3, pp. 1761-1771, March 2016. Article (CrossRef Link).

[3] H.V. Poor and O. Hadjiliadis, Quickest detection, Cambridge: Cambridge University Press, 2009.

[4] L. Lai, Y. Fan, and H.V. Poor, "Quickest detection in cognitive radio: A sequential change detection framework," in Proc. of Global Telecommunications Conference, 2008. IEEE GLOBECOM 2008. IEEE, pp. 1-5, 2008. Article (CrossRef Link).

[5] Junrong Gu, Sung Jeen Jang and Jae Moung Kim, "A Proactive Dynamic Spectrum Access Method against both Erroneous Spectrum Sensing and Asynchronous Inter-Channel Spectrum Sensing[J]," KSII Transactions on Internet and Information Systems, vol. 6, no. 1, pp. 361-378, 2012. Article (CrossRef Link).

[6] X. Zhu, B.Champagne, W.P. Zhu, "Rao test based cooperative spectrum sensing for cognitive radios in non-Gaussian noise,” Signal Processing, vol. 97, pp. 183-194, 2014.

Article (CrossRef Link).

[7] C.Yao, Q.Wu, "A hybrid combination scheme for cooperative spectrum sensing in cognitive radio networks," Mathematical Problems in Engineering, vol. 2014, pp. 7, 2014.

Article (CrossRef Link). 
[8] H. Li, "Cyclostationary feature based quickest spectrum sensing in cognitive radio systems," in Proc. of Vehicular Technology Conference Fall (VTC 2010-Fall), 2010 IEEE 72nd. IEEE, pp. 1-5, 2010. Article (CrossRef Link).

[9] Z.Yu, X.Chen, S.Hoyos, et al., "Mixed-signal parallel compressive spectrum sensing for cognitive radios,” International Journal of Digital Multimedia Broadcasting, vol. 2010, 2010. Article (CrossRef Link).

[10] C. Li, H. Li, and H. Dai, "Collaborative quickest detection in adhoc networks with delay constraint-part II: Multi-node network,” in Proc. of Information Sciences and Systems, 2008. CISS 2008. 42nd Annual Conference on. IEEE, pp. 600-605, 2008. Article (CrossRef Link).

[11] Quan, Liu, Jun, Gao, Yunwei, Guo, Siyang, Liu, “Attack-Proof Cooperative Spectrum Sensing Based on Consensus Algorithm in Cognitive Radio Networks[J],” KSII Transactions on Internet and Information Systems, vol. 4, no. 6, pp. 1042-1062, 2010. Article (CrossRef Link).

[12] E.S. Page, “Continuous inspection schemes” Biometrika, vol. 41, no. 1-2, pp. 100-115, 1954. Article (CrossRef Link).

[13] G. Lorden, "Procedures for reacting to a change in distribution," The Annals of Mathematical Statistics, vol. 42, no. 6, pp. 1897-1908, 1971. Article (CrossRef Link).

[14] G. Lorden, “On excess over the boundary," The Annals of Mathematical Statistics, vol. 41, no.2, pp. 520-527, 1970. Article (CrossRef Link).

[15] E.J. Candes, "The restricted isometry property and its implications for compressed sensing," Comptes Rendus Mathematique, vol. 346, no. 9-10, pp.589-592, 2008. Article (CrossRef Link).

[16] E.J. Candes, J. Romberg, and T. Tao, "Robust uncertainty principles: Exact signal reconstruction from highly incomplete frequency information," Information Theory, IEEE Transactions on, vol. 52, no. 2, pp. 489-509, 2006. Article (CrossRef Link).

[17] R. Baraniuk, "Compressive sensing," in Proc. of 2008 42nd Annual Conference on Information Sciences and Systems, pp. iv-v, 2008. Article (CrossRef Link).

[18] Y.Gao, Y.Chen, "Energy-Based Spectrum Sensing under Nonreconstruction Framework," Mathematical Problems in Engineering, vol. 2015, pp. 16, 2015. Article (CrossRef Link).

[19] S.M. Mishra, A. Sahai, R.W. Brodersen, “Cooperative sensing among cognitive radios," in Proc. of Communications, 2006. IEEE International Conference on. IEEE, pp. 1658-1663, 2006. Article (CrossRef Link).

[21] Y. Wang, G. Zhang, "Compressed wideband spectrum sensing based on discrete cosine transform,” The Scientific World Journal, vol. 2014, pp. 5, 2014. Article (CrossRef Link).

[22] Zhou L, Wu D, Dong Z, et al., "When Collaboration Hugs Intelligence: Content Delivery over Ultra-Dense Networks[J],” IEEE Communications Magazine, vol. 55, no. 12, pp. 91-95, 2017. Article (CrossRef Link).

[23] Zhou L, Wu D, Chen J, et al., "Greening the Smart Cities: Energy-Efficient Massive Content Delivery via D2D Communications[J],” IEEE Transactions on Industrial Informatics, vol. 14, no. 4, pp. 1626-1634, 2018. Article (CrossRef Link).

[24] Zhou L, Wu D, Chen J, et al., "When Computation Hugs Intelligence: Content-Aware Data Processing for Industrial IoT[J],” IEEE Internet of Things Journal, vol. 5, no. 3, 1657-1666, 2018. Article (CrossRef Link). 


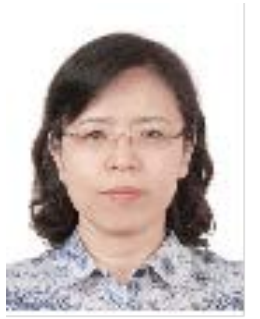

Qi Zhao received Ph.D degree in communication and information system from Beihang University, Beijing, China, in 2002. From 2002 to date, she is an associate -professor and work in Beihang University. Her current research interests include communication signal processing, image processing and cognitive radio technology.

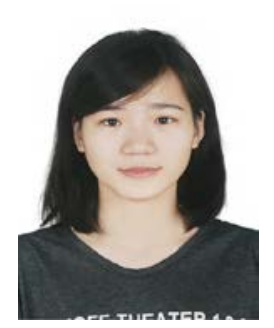

Wei Qiu received the undergraduate degree in electronic and information engineering from Beihang University, Beijing, China, in 2017. Since 2017, she has been with the School of Electronic Information Engineering, Beihang University, where she is a B.S. candidate. Her current interests include cognitive radio technology and image processing.

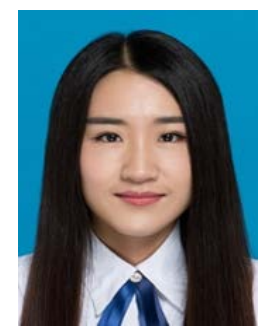

Bingqian Wang received the B.S. degree in electronic and information engineering from Beihang University, Beijing, China, in 2017. Since 2017, she has worked in AVIC Beijing Precision Engineering Institue Aircraft Industry. Her current interests include the key technologies in cognitive radio technology and Deep Learning.

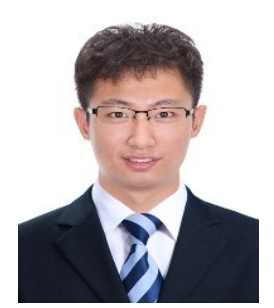

Boxue Zhang received the B.S. degree in Mathematics and System science from Beihang University, Beijing, China, in 2014. Since 2016, he has been with the School of Electronic Information Engineering, Beihang University, where he is a Ph.D. candidate. His current interests include the key technologies in the big data processing, cognitive radio technology and Deep Learning. 